\title{
Term Pregnancy after Unilateral Uterine Artery Embolization for Aggressive Uterine Arteriovenous Malformation
}

\author{
Javorka Vladimir ${ }^{1}$, Malik Miroslav ${ }^{1}$, Mizickova Magdalena ${ }^{1}$ and Korbel Miroslav ${ }^{2 *}$ \\ ${ }^{1}$ Department of Radiology, University of Hospital Bratislava, Slovak Republic \\ ${ }^{2}$ Department of Gynecology and Obstetrics, University Hospital Bratislava, Slovak Republic
}

*Corresponding author: Korbel Miroslav, Department of Gynecology and Obstetrics, University Hospital Bratislava \& University Hospital Bratislava, Slovak Republic.

To Cite This Article: Korbel Miroslav, Term Pregnancy after Unilateral Uterine Artery Embolization for Aggressive Uterine Arteriovenous Malformation. 2020 - 8(2). AJBSR.MS.ID.001255. DOI: 10.34297/AJBSR.2020.08.001255.

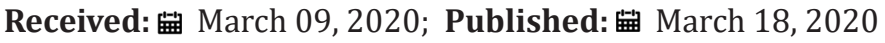

\begin{abstract}
Uterine Arteriovenous Malformation (AVM) is a potentially life-threatening condition. Uterine Artery Embolization (UAE) is a treatment method of choice for women who wish to preserve fertility. Pregnancies after UAE are at risk of perinatological complication. We described a management of uterine AVM with unilateral uterine artery embolization followed by a successful term pregnancy.
\end{abstract}

Keywords: Uterine Artery Embolization; Uterine Arteriovenous Malformation; Pregnancy; perinatological; Dilatation, Curettage; Diethylstilbestrol; Abortions; Blood Transfusion; Computer Tomography; Magnetic Resonance Imaging

\section{Introduction}

Uterine Arteriovenous Malformations (AVMs) are very rare. The first case of uterine AVM was reported in 1926 [1]. Uterine AVMs can be classified as congenital or acquired [2]. Acquired AVMs occur in reproductive women following uterine Dilatation and Curettage (D\&C), cesarean section, myomectomy, normal vaginal delivery, spontaneous abortions, and cesarean scar pregnancy. Less commonly arise from trophoblastic disease, endometrial or cervical malignancy, uterine infection or diethylstilbestrol exposure [3-5]. The accurate incidence of uterine AVMs remains unclear. Bleeding is the main symptom of AVMs and in most cases is vaginal, rarely intraperitoneal. Bleeding is in $70 \%$ of patients intermittent, in $30 \%$ massive, requiring blood transfusion [6].

Ultrasonography is preferred as a primary diagnostic method in patients with vaginal bleeding and suspected uterine AVMs [3]. Computer Tomography (CT) or Magnetic Resonance Imaging (MRI) can visualize the extent of the AVM, arterial feeders, relationships of the AVM to the uterus and to the surrounding anatomical structures [7]. Digital subtraction angiography is the gold standard technique for diagnosing uterine AVM, but currently it is used only when the embolization of uterine AVM is performed. The therapy of AVMs includes medical management of bleeding and in cases of severe hemorrhage blood transfusion, hysterectomy or UAE is performed [8-10].

\section{Case Presentation}

A 31-year-old woman, gravida 3, para 1, was admitted to our hospital due to irregular bleeding after D\&C because of Retained Product of Conception (rPOC). Her obstetrical history included term cesarean section delivery 2-years before (during pregnancy low-laying placenta was located on posterior uterine wall) and spontaneous abortion at 14 th week of pregnancy treated with D\&C (placenta was also located on posterior uterine wall). After this abortion she had 3 normal menstrual cycles and afterwards irregular vaginal bleeding started lasting 2 months with the increase of Human Chorionic Gonadotropin (hCG) level to 18,605IU/l. D\&C was performed because of rPOC. At that time normal architecture of myometrium was described on Transvaginal Ultrasonography 
(TVUS). Irregular bleeding started two weeks after D\&C and hCG level decreased to $1,279 \mathrm{IU} / \mathrm{l}$.

Uterine cavity was without rPOC on TVUS, but at lower segment of posterior uterine wall area with different echogenicity to $2.3 \mathrm{~cm}$ with AVM-Peak Systolic Velocity (PSV) $60 \mathrm{~cm} / \mathrm{s}$ was detected. CT revealed $2.2 \mathrm{~cm}$ uterine hyper vascular lesion. During next 4 weeks hCG level decreased to 23IU/l, but AVM on TVUS progressed up to
$4.7 \mathrm{~cm}$, on color Doppler imaging hypervascularity and turbulent flow with PSV $43 \mathrm{~cm} / \mathrm{s}$ was detected. Because of intermittent scanty bleeding, high risk of heavy bleeding and woman's fertility preservation desire, UAE was offered. The embolization was performed 8 weeks after D\&C. Through right transfemoral access we catheterized the Left Internal Iliac Artery (LIIA) and the Left Uterine Artery (LUA), for catheterization reshaped 5F catheter RUC (Roberts Uterine Curve) (COOK, Wiliam Cook Europe, Bjaeverskov, DK) was used.

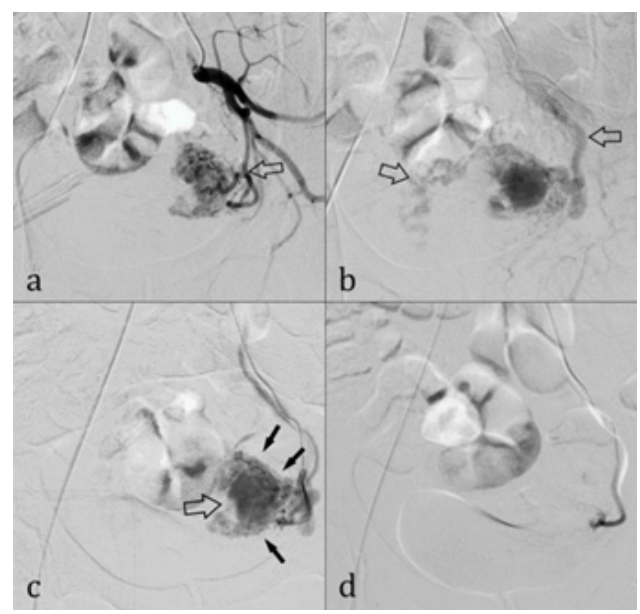

Figure 1: a) Selective arteriography of the left internal iliac artery, arterial phase. Enlarged diameter of left uterine artery (arrow) feeding AVM. b) Selective arteriography of the left internal iliac artery, early venous phase. The AVM is drained into the both internal iliac veins (arrows). c) Superselective arteriography of the left uterine artery. Multiple tortuous vessels (small arrows) and a large lacune (empty arrow) are present in the AVM. d) Superselective arteriography of the left uterine artery after embolization with gelatine foam and PVA particles, the AVM is totally occluded.

Wide diameter LUA with large AVM on the left side of pelvis and rapid drainage into the both internal iliac veins was found on the performed angiography (Figure $1 \mathrm{a} \& 1 \mathrm{~b}$ ). Then the distal course of LUA was catheterized with microcatheter $(0.025$ "System Micro Catheter, Progreat, Terumo Europe, N.V., Leuven, Belgium) and the embolization of the AVM was performed. The microparticles PVA500, size 500-710 $\mathrm{m}$ (COOK, William Cook Europe, Bjaeverskov, DK) and gelatin foam slurry mixture (Gelita-Spon ${ }^{\circledR}$ Standard, Gelita Medical, Eberbach, Germany) were used as an embolization material. The embolization of LUA was stopped when the AVM appeared to be totally occluded (Figure 1c \& 1d). After the completion of the LUA embolization we catheterized the Right Uterine Artery (RUA) and super selective angiography of the RUA was performed.

No feeding arteries from RUA to AVM was found, the same situation persisted on repeated angiography ten minutes later, so we decided to finish the procedure. Patient the day after UAE was painless and vascularity on TVUS color Doppler imaging into AVM was absent. Eleven days after UAE the hCG level was negative and normal menstrual cycle started a 1 month after UAE. Five months after UAE myometrial structure on TVS was normal, without vascularity. Nineteen months after UAE the patient became pregnant (location of placenta during pregnancy was on posterior uterine wall). Repeated cesarean section was done 28 months after UAE because of bleeding from partial placental abruption, and a healthy girl 2,880gr, 49cm, Apgar score 10/10 at 38+1-week pregnancy was delivered. Five years after UAE and 3 years after birth, patient have normal regular menstrual cycle.

\section{Discussion}

In the past, hysterectomy was the main therapeutic method for bleeding uterine AVMs. The first successful embolization of symptomatic uterine AVMs has provided a less invasive treatment option [11]. The issue of fertility and gravidity after UAE treatment of fibroid, as well as AVMs, is a discussed topic. Several complications during perinatal period and the effect of UAE on fertility have been described. The risk of impaired fertility and perinatological complications is associated with the possible atrophy of endometrium as a result of extensive embolization. Possible cause is endometrial ischemia caused by UAE. Atrophy of endometrium with amenorrhea after UAE was reported [12]. More frequent occurrence of pre-eclampsia, placental abnormalities (abnormally invasive placenta), fetal growth restriction and premature delivery as a result of hypoperfusion of endometrium are other complications after UAE [13].

Conversely, other studies claim that UAE is a safe and effective method of uterine fibroid treatment in patients planning pregnancy [14]. But several case reports and case series described placental abnormality (placenta previa, abnormally invasive placenta), 
placental abruption and premature delivery also after UAE due to AVMs $[15,16]$. In previously published papers, various embolic agents and their combinations such as glue, gelatin foam, coils, PVA particles, NBCA, Onyx and microspheres have been used for UAE in patients with AVMs. Given the rare incidence of uterine AVMs and the low number of embolization procedures performed, with heterogeneity of the embolic agents used, we have no good evidence of which one is the most effective. In most clinical instances, vessel recanalization after gelatin foam embolization was seen. Gelatin foam provides vessel occlusion typically lasting 3-6 weeks, but in rare instances can lead to permanent occlusion [17].

In our case report, the patient desired fertility preservation. Decision for embolization was made due to high risk of heavy bleeding from progressive enlargement of AVMs despite hCG (trophoblastic cell activity) regression. The main goal of the embolization was to achieve complete occlusion of AVM and to minimize damage to normal vascularization of uterus. To perform embolization as minimally invasive as possible, at first, we injected a 500-710 $\mu \mathrm{m}$ PVA particles followed by gelatin slurry mixture into the LUA. The complete disappearance of pathological vascularization was achieved immediately, also documented on TVUS color Doppler the day after the procedure. The decision for unilateral embolization was based on the angiographic finding of one feeding artery from the left side and the absence of pathological vascularization from the right uterine artery. We supposed that, similarly to fibroid treatment, unilateral embolization of AVMs could lead to reduction of endometrial ischemia and potential consequent complications in pregnancy and delivery.

Generally, in most cases the UAE in the treatment of uterine fibroid or AVMs is performed bilaterally. Conclusions from several large studies strongly recommend bilateral embolization which has better clinical outcomes and less percentage of treatment failure versus unscheduled unilateral embolization [18]. In cases of scheduled unilateral embolization, an enough clinical effect was achieved, when the fibroid was dominantly supplied by one uterine artery [19]. Unlike the arterial supply of uterine fibroids, the arterial supply of acquired AVMs after trauma or gynecological interventions is characterized by a single arteriovenous communication between the branches of uterine artery and the myometrial venous plexus. This fact could explain a high success rate in cases of unilateral UAE in the treatment of acquired uterine AVMs [10].Recent published data (but from small series) conclude, that the UAE for uterine AVMs is an effective and safe technique which does not impair fertility and obstetric prognosis is also good. UAE is recommended as the first-line uterine AVMs modality treatment for women planning pregnancy $[20,21]$.

\section{Conclusion}

Endovascular embolization of uterine AVMs is an effective treatment with minimal complications and for patients who desire fertility preservation is the method of choice. In our case, a complete regression of acquired uterine AVM was achieved after unilateral UAE and the patient gave birth to a healthy child 28 months following the procedure. Due to rare incidence of this pathology, it is not yet possible to propose the optimal protocol for endovascular embolization.

\section{References}

1. Dubreuil G, Loubat E (1926) Aneurysm circus de l'uterus. Ann Anat Pathol 3: 697-718.

2. Robert MO, David L, Rumsey C (2015) Uterine arteriovenous malformation, images, and management. Mil Med 180(1): 177-180.

3. Timor Tritsch IE, Haynes MC, Monteagudo A, Khatib N, Kovács S (2016) Ultrasound diagnosis and management of acquired uterine enhanced myometrial vascularity arteriovenous malformations. Am J Obstet Gynecol 214(6): 1-10.

4. Khan S, Saud S, Khan I, Achakzai B (2019) Acquired uterine arteriovenous malformation following dilatation and curettage treated with bilateral uterine artery embolization: A Case Report. Cureus 11(3): 4250.

5. Szpera GA, Gruca SK, Bręborowicz GH, Ropacka LM (2018) Uterine arteriovenous malformation-diagnosis and management. Ginekol Pol 89(5): 276-279.

6. Lowenstein L, Solt I, Deutsch M, Kerner H, Amit A (2004) A Lifethreatening event: uterine cervical arteriovenous malformation. Obstet Gynecol 103(5 pt 2): 1073-1075.

7. Gulati MS, Paul SB, Batra A, Sarma D, Dadhwal V, et al. (2000) Uterine arteriovenous malformations: the role of intravenous'dual-phase' CT angiography. Clin Imaging 24(1): 10-14.

8. Vilos AG, Oraif A, Machado M, Abu RB, Vilos GA (2018) Resolution of uterine arteriovenous malformation and maintenance of reproduction in 20 women treated with a GnRH agonist concomitantly with an aromatase inhibitor and tranexamic acid. J Obstet Gynaecol Can 41(6): 772-781.

9. Vilos AG, Vilos GA, Hollet CJ, Rajakumar C, Garvin G, et al. (2015) Uterine artery embolization for uterine arteriovenous malformation in five women desiring fertility: pregnancy outcomes. Hum Reprod 30(7): 1599-1605.

10. Yoon DJ, Jones M, Taani JAl, Buhimschi C, Dowell JD (2016) A Systematic review of acquired uterine arteriovenous malformations: pathophysiology, diagnosis, and transcatheter treatment. Am J P Rep 6(1): 6-14.

11. Wilms GE, Favril A, Baert AL, Poppe W, Van Assche AF (1986) Transcatheter embolization of uterine arteriovenous malformations. Cardiovasc Intervent Radiol 9(2): 61-64.

12. Tropeano G, Litwicka K, Stasi CD, Romano D, Mancuso S (2003) Permanent amenorrhea associated with endometrial atrophy after uterine artery embolization for symptomatic uterine fibroids. Fertil Steril 79(1): 132-135.

13. Klatsky PC, Tran ND, Caughey AB, Fujimoto VY (2008) Fibroids and reproductive outcomes: a systematic literature review from conception to delivery. Am J Obstet Gynecol 198(4): 357-366.

14. Pisco JM, Duarte M, Bilhim T, Branco J, Cirurgião F, et al. (2017) Spontaneous pregnancy with a live birth after conventional and partial uterine fibroid embolization. Radiology 285(1): 302-310.

15. Soeda S, Kyozuka H, Suzuki S, Yasuda S, Nomura Y, et al. (2014) Uterine artery embolization for uterine arteriovenous malformation is associated with placental abnormalities in the subsequent pregnancy: two cases report. Fukushima J Med Sci 60(1): 86-90.

16. Vilos AG, Vilos GA, Vrijer BD, Kozak R, Abu RB (2010) Fertility and pregnancy outcomes following Uterine Artery Embolization (UAE) for uterine Arteriovenous Malformation (AVM). Gynecol Surg 7: 401-405. 
17. Medsinge A, Zajko A, Orons P, Amesur N, Santos E (2014) A casebased approach to common embolization agents used in vascular Interventional Radiology. AJR 203(4): 699-708.

18. Spies JB, Myers ER, Worthington KR, Mulgung J, Goodwin S, et al. (2005) The FIBROID registry: symptom and quality of life status 1 year after therapy. Obstet Gynecol 106(6): 1309-1318.

19. Stall L, Lee J, McCullough M, Nsrouli MH, Spies James B (2011) Effectiveness of elective unilateral uterine artery embolization: a casecontrol study. J Vasc Interv Radiol 22(5): 716-722.
20. Delplanque S, Le Lous M, Proisy M, Joueidi Y, Bauville E, et al. (2019). Fertility, pregnancy, and clinical outcomes after uterine arteriovenous malformation management. J Minim Invasive Gynecol 26(1): 153-161.

21. Guida M, Maffucci D, Iannuzzi G, Giordano M, Luciano G, et al. (2018) Successful pregnancy after uterine artery embolization for uterine arterovenous malformation: a rare case report. Int J Womens Health 10: $745-750$. 